\title{
Formar, enseñar y aprender sobre la práctica científica en primaria con un juego de cajas negras
}

\author{
María-Antonia Manassero-Mas ${ }^{1}$ (D) Antoni-J. Bennàssar-Roig ${ }^{2}$ (D) y Ángel Vázquez-Alonso ${ }^{3}$ \\ Artículo de innovación. Recibido: 21/10/2020. Aceptado: 02/03/2021. Publicación avanzada: 05/05/2021. Publicado: 01/07/2021
}

\section{Resumen}

INTRODUCCIÓN. Aprender cómo funciona la comunidad científica para validar conocimientos es un componente esencial de la alfabetización científica que no se enseña adecuadamente por su complejidad cognitiva, la carencia de recursos didácticos y la insuficiente atención a estos temas en la formación del profesorado. El objetivo es innovar la formación y el desarrollo profesional del profesorado mediante juegos serios (cajas negras) que incentiven esta enseñanza epistémica y su aprendizaje por alumnado en primaria.

MÉTODO. Tres maestros y sus estudiantes participan en un proceso metodológicamente mixto: desarrollo del juego como recurso didáctico, intervención educativa con estudiantes e investigación-acción sobre la práctica docente de los maestros.

RESULTADOS. Se presentan como resultados, las actividades, los productos y el desarrollo profesional docente por medio del estudio de la lección: planificación de la secuencia didáctica, producciones del alumnado y evaluación de la formación (maestros) y de la motivación e interés de maestros y alumnado. La eficacia del juego se justifica por la valoración positiva de los docentes y la realización, aceptación y motivación hacia las tareas del alumnado.

DISCUSIÓN. Se discuten los logros, los límites y las implicaciones futuras en relación con los objetivos de la experiencia tanto para la educación científica como para desarrollar en las escuelas la sostenibilidad de la experiencia de formación con juegos epistémicos.

\section{Palabras clave}

Educación científica; Formación de profesorado; Práctica científica; Juego educativo; Enseñanza basada en investigación.

Datos de los autores

${ }^{1}$ Universidad de las Islas Baleares, España. Departamento de Psicología, Catedrática de Universidad.

${ }^{2}$ Universidad de las Islas Baleares, España. Departamento de Biología, Catedrático de Escuela Universitaria.

${ }^{3}$ Universidad de las Islas Baleares, España. Centro de Estudios de Posgrado, Investigador colaborador. Correo para la correspondencia: angel.vazquez@uib.es

Referencia recomendada

Manassero-Mas, M.A., Bennàssar-Roig, A.J., y Vázquez-Alonso, A. (2021). Formar, enseñar y aprender sobre la práctica científica en primaria con un juego de cajas negras. REIRE Revista d'Innovació i Recerca en Educació, 14(2), 1-22.

https://doi.org/10.1344/reire2021.14.232607

(C) 2021 Los autores. Este artículo es de acceso abierto sujeto a la licencia Reconocimiento 4.0 Internacional de Creative Commons, la cual permite utilizar, distribuir y reproducir por cualquier medio sin restricciones siempre que se cite adecuadamente la obra original. Para ver una copia de esta licencia, visite https://creativecommons.org/licenses/by/4.0/ 


\section{Títol (català)}

Formar, ensenyar i aprendre sobre la pràctica científica a primària amb un joc de caixes negres

\section{Resum}

INTRODUCCIÓ. Aprendre com funciona la comunitat científica per validar els coneixements és un component essencial de l'alfabetització científica, que no s'ensenya adequadament a causa de la seva complexitat cognitiva, la manca de recursos didàctics i la insuficient atenció en aquests temes a la formació del professorat. L'objectiu d'aquest article és ajudar a innovar la formació i el desenvolupament professional del professorat mitjançant jocs seriosos (caixes negres) que incentivin aquest ensenyament epistèmic i el seu aprenentatge per part de l'alumnat de primària.

MÈTODE. Tres mestres i els seus estudiants participen en un procés metodològicament mixt: desenvolupament del joc com a recurs didàctic, intervenció educativa amb estudiants i investigació-acció sobre la pràctica docent amb mestres.

RESULTATS. Es presenten com a resultat les activitats, els productes i el desenvolupament professional docent mitjançant l'estudi de la lliçó: planificació de la seqüència d'ensenyament, produccions de l'alumnat, i avaluació de la formació (mestres) i de la motivació i interès de mestres i estudiants. L'eficàcia del joc com a recurs es justifica per la valoració positiva que en fan els docents i per la realització, acceptació i motivació de l'alumnat vers les tasques.

DISCUSSIÓ. Es discuteixen els assoliments, els límits i les implicacions futures en relació amb els objectius de l'experiència, tant pel que fa a l'educació científica com per desenvolupar a les escoles la sostenibilitat de l'experiència de formació amb jocs epistèmics.

Paraules clau

Educació científica; Formació de professorat; Pràctica científica; Joc educatiu; Ensenyament basat en investigació.

\section{Title (English)}

Training, teaching and learning scientific practices at primary school with a Black Box game

\section{Abstract}

INTRODUCTION. Learning how science and scientists work to validate knowledge is an essential component of scientific literacy. Because of its cognitive complexity, the lack of educational resources, and the insufficient teacher training on this issue, this skill is often inadequately taught. This study aims to innovate primary teachers' training and professional development by introducing serious games (black boxes) that encourage this teaching and the primary students' epistemic learning.

METHOD. Three teachers and their students took part in a mixed-method process: playing the game as a pedagogical resource, as an educational intervention with students, and as an action-research on teaching practices with primary teachers.

RESULTS. The results display the activities, products and teachers' professional development in the lesson under study: the planning of the teaching sequences, the productions of students' learning, teachers' and students' evaluation of the training, and their motivation and interest regarding the whole experience. The effectiveness of the game is borne out by the teachers' positive assessment and the students' engagement and motivation in doing the learning tasks.

DISCUSSION. We discuss the achievements, limits and future implications in relation to the objectives of the experience, both for science education in general and for promoting the sustainability of training experiences with epistemic games in the school setting.

Keywords

Science education; Teacher training; Scientific practice; Educational game; Research-based teaching. 


\section{Introducción}

El objetivo perenne de la educación científica es la alfabetización, que actualmente se interpreta como "ciencia para todos» inclusiva, capaz de preparar adecuadamente a la ciudadanía para la vida cotidiana en un mundo cada vez más sostenible y permeado por la ciencia y tecnología, en contraposición a la tradicional "ciencia para científicos» (Hodson, 2008; Millar, 2006; Vázquez-Alonso y Manassero-Mas, 2018). La alfabetización científica tiene dos componentes básicos: uno cognitivo, formado por conceptos, hechos, principios y procesos de la ciencia, que tradicionalmente dominan los libros de texto y currículos escolares; y otro componente formado por los innovadores conocimientos "sobre» la ciencia, que a menudo se denominan también naturaleza de la ciencia (en didáctica de la ciencia) o conocimiento epistémico (en la educación general). Este conocimiento innovador trata sobre cómo la ciencia y la comunidad científica validan el conocimiento o intervienen en la sociedad, y constituye el contenido de referencia de este estudio (Roberts, 2007; Vázquez-Alonso y Manassero-Mas, 2012).

Los conocimientos de naturaleza de la ciencia/conocimientos epistémicos (en lo sucesivo $\mathrm{NdC} / \mathrm{CE}$ ) comprenden un conjunto de meta-conocimientos complejos, multifacéticos, interdisciplinares, evolutivos y cambiantes sobre las prácticas científicas (y tecnológicas), que la historia, filosofía y sociología de la ciencia (y otras) han aportado cuando analizan las prácticas científicas y tecnológicas; entre otros, se refieren a su funcionamiento, métodos, valores, comunidad científica, relaciones entre ciencia y tecnología y con la sociedad, etc. (Lederman, 2007; NGSS, 2013; Vázquez-Alonso y Manassero-Mas, 2012). Actualmente, hay acuerdo en la comunidad investigadora acerca de la necesidad de innovar la educación científica enseñando conocimientos "sobre" la ciencia, como elementos claves de la alfabetización; paralelamente, hay un debate acerca de los contenidos más apropiados de esa enseñanza y que se manifiesta en el uso de denominaciones diversas, tales como naturaleza de la ciencia, ideas sobre la ciencia, rasgos de la ciencia, ciencia total, entre otras (Vázquez-Alonso y Manassero-Mas, 2012; Vesterinen et al., 2014). La denominada visión de consenso propone una lista reducida de temas, argumentando la potencial funcionalidad docente de lo simple (Abd-el-Khalick y Akerson, 2009; Lederman, 2007; McComas, 2008); otros sugieren visiones más amplias, porque una lista simple supone una reducción y devaluación conceptual del constructo (Acevedo-Díaz y García-Carmona, 2016; Allchin, 2011; Matthews, 2012; Osborne et al., 2003, entre otros).

Las re-conceptualizaciones de Erduran y Dagher (2014) y Manassero y Vázquez (2019) analizan el conocimiento epistémico ( $\mathrm{NdC} / \mathrm{CE}$ ) y coinciden en describirlo con base en dos amplias dimensiones, una cognitivo-epistemológica y otra social-institucional; el segundo trabajo las desarrolla en una taxonomía abierta y flexible donde se estructuran decenas de temas y subtemas. En la misma línea, el programa de educación científica para las nuevas generaciones (NGSS, 2013) integra en el concepto de práctica científica, de forma natural, la pluralidad de prácticas científicas e ingenieriles expuestas en esas reconceptualizaciones.

La enseñanza y el aprendizaje del conocimiento NdC/EC sobre la práctica científica no es fácil y constituye aún una innovación pendiente en la educación científica. Por el lado metodológico, diversas investigaciones han allanado el camino al mostrar que los enfoques más eficaces involucran planteamientos explícitos de la enseñanza y actividades de reflexión meta-cognitiva para el alumnado (Abd-el-Khalick y Akerson, 2009; Deng et al., 2011; García-Carmona et al., 2011; Lederman, 2007). El enfoque explícito y reflexivo también se ha demostrado eficaz para mejorar la comprensión del alumnado de primaria en algunos aspectos (cambio en ciencia), aunque no tanto en otros, como la distinción observación-inferencia, el carácter 
subjetivo o los aspectos sociales (Akerson y Donnelly, 2010; Akerson et al., 2014; Khadija et al., 2015; Quigley et al., 2011).

La literatura apunta unánimemente al principal inconveniente para una enseñanza eficaz de $\mathrm{NdC} / \mathrm{CE}$ : el profesorado no tiene una adecuada comprensión de esos temas (García-Carmona et al., 2011; Lederman, 2007). Además, los estudios relativos a la educación primaria son más escasos en la literatura; la revisión de Cofré et al. (2019) reseña solo nueve estudios entre 2010-2018 sobre la comprensión y formación de profesores de primaria en ejercicio, y, como instrumento de formación de los profesores recomienda la planificación y aplicación de las clases de enseñanza de NdC/CE; Akerson et al. (2019) sostienen que aplicar estrategias de interacción en clase y trabajo escrito del alumnado también es eficaz para que los maestros enseñen y los alumnos aprendan NdC. Estas perspectivas se adoptan en este estudio.

\subsection{Juegos educativos serios}

Desde la primera infancia todas las actividades del niño para conocer y acomodarse en su entorno tienen un sentido lúdico y funcional, de modo que el juego es esencial para el desarrollo integral en toda la infancia. Los juegos se caracterizan por el planteamiento de retos y unas reglas para conseguirlos, que regulan las interacciones de los jugadores para entretenerse, competir y ganar. Los juegos se suelen clasificar en cinco categorías: físicos, con objetos, simbólicos, dramáticos y reglados. En el juego con objetos, el jugador actúa como un científico explorando objetos y los juegos reglados pueden aportar el desarrollo de los aspectos siguientes: actividad, entretenimiento, significatividad, interactividad, inhibición de la realidad, diversión, control del jugador, curiosidad, desafío, contenidos explícitos y normas (Whitebread, 2012).

El entretenimiento es un aspecto asociado a cualquier juego, pero cuando el entretenimiento no es el fin, sino sólo un medio para lograr otros fines de mayor calidad, tales como formación, aprendizaje, salud, comunicación, etc. el juego se considera un juego serio. Los juegos serios centrados en el aprendizaje ofrecen a los jugadores información nueva, cambios de comportamientos o habilidades nuevas, y, por eso, algunos autores los denominan también juegos para el aprendizaje lúdico (Hassinger-Das et al., 2017).

Diversos estudios muestran que los juegos serios son más eficaces que la instrucción convencional para producir cambios conductuales, cognitivos y meta-cognitivos. El meta-análisis sobre el aprendizaje visible de Hattie (2009) asigna a los juegos y videos interactivos un relevante tamaño de efecto medio sobre el aprendizaje $(d=0,50-0,52)$ que justifica el uso de juegos como recursos de enseñanza. La revisión de varios meta-análisis de Clark et al. (2016) indica que los juegos serios digitales se asocian a una mejora media moderada $(d=0,33)$ para diversas variables cognitivas, actitudinales, conocimientos, auto-eficacia, procedimientos y retención respecto a los aprendizajes sin juegos, que está en línea también con el estudio de Wouters et al. (2013). Además, los juegos añaden otras ventajas educativas: los estudiantes no necesitan conocimientos previos para jugar y el diseño de sus contenidos es muy abierto y flexible, lo cual permite adaptarlos a las características del estudiante y al desarrollo de los aprendizajes cooperativos y sociales (McGonigal, 2011).

Todas estas características han causado un gran desarrollo de los juegos serios en todas las disciplinas y también en ciencias, aunque mayoritariamente enfocados a enseñar los contenidos cognitivos, mientras los juegos enfocados al aprendizaje del conocimiento epistémico son escasos. Por ejemplo, el análisis de 31 investigaciones (2000-2011) que aplicaron juegos digitales de 
contenido científico muestra que ningún juego abordó el aprendizaje de temas de naturaleza de la ciencia (Li y Tsai, 2013). No obstante, ya se ha indicado que los juegos con objetos favorecen e impulsan en los jugadores el rol de científicos (Whitebread, 2012), y, en particular, los juegos que simulan problemas permiten enseñar también aspectos epistémicos complejos, tales como la conducta y el pensamiento de la comunidad científica, especialmente la argumentación con base en pruebas, piedra angular de la ciencia (Gee, 2007; Squire, 2011).

En este sentido, los juegos de cajas negras se eligen como material didáctico aquí porque ofrecen una analogía realista de las prácticas científicas (responder una pregunta en un contexto de información limitada) y permiten la emulación de la conducta, las habilidades y el pensamiento de la comunidad científica, que los hace idóneos para el aprendizaje lúdico de los complejos aspectos $\mathrm{NdC} / \mathrm{CE}$. Incluso permiten simular principios epistemológicos contrapuestos de la práctica científica explícitamente, tales como la creatividad y el respeto a las reglas, o en la dimensión social, cooperar y a la vez competir, en la medida que el juego exige someterse a las reglas, pero también rivalizar para intentar ganar (Vázquez-Alonso y Manassero-Mas, 2017).

La justificación del diseño educativo y aplicación de cajas negras proviene de su capacidad para abordar las principales dificultades de la enseñanza de NdC/CE: suplir la carencia de recursos, hacer fácil y motivador un aprendizaje difícil para estudiantes tan jóvenes y ofrecer formación sencilla y útil para el profesorado (enseñanza de un tópico relegado). Además, la flexibilidad del juego agrega ventajas para su uso en primaria, pues permite al profesorado adaptar fácilmente su diseño al desarrollo, evolución y necesidades del alumnado, porque primaria ha sido un contexto poco frecuente de las investigaciones sobre enseñanza de NdC/CE y porque algunos estudios demuestran la capacidad de este alumnado para aprender aspectos epistémicos (Akerson y Donnelly, 2010; Cofré et al., 2019; Quigley et al., 2011).

\section{Objetivos}

Esta propuesta innovadora de enseñanza del conocimiento NdC/CE se plantea como objetivos algunas carencias actuales del tópico. El primer objetivo es la mejora del desarrollo profesional y formación del profesorado, a través de la apropiación didáctica del juego como un recurso para enseñar el tópico innovador de NdC/CE (Acevedo-Díaz y García-Carmona, 2016) y el progreso en habilidades de pensamiento científico de los docentes, mediante el diseño del plan de lecciones para lograr el aprendizaje explícito y reflexivo del alumnado. El segundo objetivo plantea el reto educativo de aplicar un recurso innovador (juego de cajas negras), porque apenas ha sido usado para enseñar $\mathrm{NdC/CE}$ y pensamiento científico. El tercer objetivo afrontar el estudio de $\mathrm{NdC} / \mathrm{CE}$ en el nivel de primaria, poco frecuente en la literatura. El cuarto objetivo, común a docentes y estudiantes, es el desarrollo de habilidades de pensamiento científico y crítico mediante su práctica activa en la lección de $\mathrm{NdC} / \mathrm{CE}$, porque son relevantes para la educación científica y transversales para otras disciplinas y la vida cotidiana (Vázquez-Alonso y Manassero-Mas, 2018). Las habilidades de pensamiento en este objetivo son:

- Hacer preguntas y definir problemas.

- Analizar e interpretar datos.

- Construir explicaciones y diseñar soluciones. 
- Participar en la argumentación con base en pruebas

- Desarrollar y usar modelos.

- Planificar y realizar investigaciones.

- Obtener, evaluar y comunicar información.

Estas habilidades se basan en los siguientes supuestos de pensamiento científico: la ciencia supone orden y consistencia en los sistemas naturales, la investigación científica utiliza una variedad de métodos, el conocimiento científico (leyes, modelos y teorías) explica los fenómenos naturales con base en pruebas empíricas y permanece abierto a la revisión (provisionalidad), desde nuevas pruebas y hechos, que pueden sugerir cambios.

En suma, la literatura informa que enseñar NdC/CE es un tema relevante de la didáctica de la ciencia, pero aún pendiente y abierto por los grandes obstáculos que afronta: la falta de formación de los docentes y de recursos educativos apropiados, junto con la ausencia de tradición y la presencia de numerosas ideas ingenuas sobre el tema; estas carencias se acentúan más en los niveles elementales, por la precaria formación científica de los maestros. El objetivo de este estudio es afrontar estas carencias, mediante un recurso didáctico innovador (juego de cajas negras), en una experiencia de formación de maestros de primaria sobre $\mathrm{NdC} / \mathrm{CE}$ en la acción práctica: el maestro desarrolla el plan de la lección, basado en el juego de cajas negras, y lo aplica en el aula para verificar su eficacia en el aprendizaje de la práctica científica por el alumnado; la intervención con el juego plantea el desempeño por el alumnado del rol de comunidad científica, lo cual implica estimular el pensamiento creativo y argumentativo y generar ideas y modelos mediante reflexión, interacción y cooperación.

La pregunta de investigación es: ¿el juego serio de cajas negras es eficaz como formación de docentes de primaria para enseñar sobre la práctica científica? Además, como enseñanza y aprendizaje van unidos, otra pregunta complementa la anterior: ¿la aplicación del juego en el aula es eficaz para desarrollar los aprendizajes y el pensamiento del alumnado?

\section{Método}

Se centra en la experiencia de formación de maestros sobre conocimientos epistémicos y se corresponde con una metodología cualitativa de investigación-acción, donde los docentes investigan sobre su propia práctica con el juego serio de cajas negras: planifican, aplican, participan y evalúan su enseñanza y el aprendizaje de los estudiantes (Vázquez et al., 2017).

\subsection{Participantes}

Los participantes en esta actividad son tres maestros de primaria en ejercicio (dos hombres y una mujer, entre 13 y 19 años de docencia), cuya formación inicial es generalista y carente de formación específica de ciencias. Ejercen en tres escuelas situadas en la periferia de una gran ciudad, en una ciudad media y en una población turística costera, siendo tutores de dos grupos de 5. curso (21 alumnos y 25 alumnos) y de 6. curso (18 alumnos) de primaria (11-12 años), donde desarrollaron las actividades del juego de cajas negras, con un promedio de cuatro horas de actividad de aula. 
Su formación consistió en compartir unas orientaciones sobre $\mathrm{NdC} / \mathrm{CE}$, los materiales y objetivos de la actividad educativa con cajas negras para enseñar sobre la práctica científica, como parte del desarrollo del currículo de ciencias de primaria (bloque 1, la actividad de los científicos). Cada maestro personalizó el diseño de su propio estudio de lección, planificando, adaptando y aplicando el juego al contexto particular de sus estudiantes, lo que resultó en tres lecciones y experiencias diferentes, cuyos resultados y productos fueron más variados que la reducida muestra ofrecida aquí, debido a las limitaciones de espacio.

\subsection{Instrumentos}

El modelo teórico de la caja negra (llamada así porque su interior es opaco) ha sido desarrollado por académicos, como un sistema abierto que responde a estímulos externos y basado en el supuesto que existe una relación (causal) entre estímulos y respuestas observables; su uso está centrado en las relaciones entradas/salidas, pues su interior es opaco (Bunge, 1963). La caja negra conecta con otras disciplinas y la vida diaria, porque muchos dispositivos (computadores) y sistemas (cerebro) son ejemplos prácticos de cajas negras.

Los juegos con cajas negras integran características propias de los juegos con objetos (observación), los juegos simbólicos (uso del lenguaje para razonar y argumentar) y los juegos reglados (Whitebread, 2012). Por ello, las cajas negras permiten el abordaje explícito del conocimiento $\mathrm{NdC/CE}$ y del pensamiento cooperativo y reflexivo de maestros y estudiantes (emulando a la comunidad científica) y, además, la posibilidad de elaborar el juego como una experiencia integral de práctica científica (proyecto, basado en investigación o evidencias), aunque sin vinculación con el laboratorio tradicional (Vázquez-Alonso y Manassero-Mas, 2012).

Algunos estudios muestran diversas versiones de cajas negras, tales como estructuras internas (Raviolo et al., 2010), tubos con líquidos (MUSE, 2002), combinaciones de agujeros (Cartier, 2000), o simples cajas cerradas con objetos (Solís et al., 2015). En estas referencias las cajas se usan para enseñar otros conocimientos, mientras el objetivo innovador aquí es enseñar $\mathrm{NdC} / \mathrm{CE}$, conocimiento más difícil por el alto nivel cognitivo de las destrezas científicas implicadas. En este estudio, el instrumento innovador, por su aplicación a $\mathrm{NdC} / \mathrm{CE}$, es el juego de cajas negras, elegido tras una revisión de diversos trabajos entre juegos de rompecabezas, cubos, escenarios, cajas negras, tarjetas, etc. en función de satisfacer los criterios del marco teórico, tales como afrontar explícitamente un tema epistémico, permitir el desarrollo del pensamiento, la cooperación y la discusión argumentada entre los participantes y ser adaptado para primaria. El formato digital fue descartado porque no facilita tanto las interacciones cooperativas para ejercitar la construcción social del conocimiento y porque los tecnicismos, los programas y la orientación individualista podrían distraer del objetivo didáctico anterior (Vázquez-Alonso y Manassero-Mas, 2017).

La caja negra de este estudio es una caja opaca con objetos dentro; los estudiantes tratan de averiguar el número y naturaleza de los objetos, manipulando la caja para probar sus hipótesis y conjeturas. Esta sencilla y desafiante actividad introduce suavemente al alumnado en complejos principios epistémicos propios de las prácticas científicas para validar conocimientos, que aplican destrezas y procesos de pensamiento de alto nivel, con énfasis en el razonamiento basado en pruebas a través de la discusión cooperativa en grupo (Gee, 2007; Squire, 2011). Las destrezas específicas de pensamiento científico en juego son observar, descubrir, verificar, razonar sobre las observaciones, resolver objeciones y anomalías, hacer hipótesis sobre la caja y verificar las 
predicciones compatibles con las observaciones. Una exigente regla del juego consiste en permitir solo las propuestas que van acompañadas de algún argumento o razonamiento basado en pruebas; no obstante, una ventaja crucial de las cajas negras como instrumento didáctico es su flexibilidad y apertura: se pueden acomodar a las necesidades del alumnado y a varios niveles de dificultad.

\subsection{Procedimientos}

El diseño de investigación cualitativa se centra en la innovación de poder formar al profesorado de primaria y de enseñar conocimiento $\mathrm{NdC} / \mathrm{CE}$ a los estudiantes. La formación de los maestros comprende una sesión de orientación inicial con los investigadores sobre esta innovación y el instrumento de cajas negras; los maestros preparan un plan de lecciones sobre la caja negra, que implica tareas de adaptar contenidos epistémicos para su alumnado, diseñar su infusión en el currículo (momento, duración, conexiones), liderar la enseñanza en el aula, guiar y recoger los datos de las reflexiones y argumentos de los alumnos con los organizadores-guía y ayudar a los alumnos a sacar conclusiones de las actividades epistémicas. El plan de investigación-acción se completa con una sesión conjunta donde los docentes comparten sus experiencias, reflexionan, analizan y evalúan su propia eficacia en la planificación y ejecución de las actividades realizadas, los resultados obtenidos y el impacto en el aprendizaje de sus estudiantes.

La idea epistémica clave del proyecto con cajas negras es lograr que los estudiantes asuman el rol de científicos y practiquen socialmente las normas y procesos de la ciencia y las destrezas científicas de argumentación, planificación, reflexión, cooperación y gestión de las actividades en la búsqueda de conocimientos. Los docentes diseñan para sus estudiantes organizadores-guías que recogen y documentan los datos cualitativos de observaciones, reflexiones, argumentos y conclusiones de las actividades, como parte del desarrollo curricular de la secuencia de enseñanza y aprendizaje (SEA) (Figura 1).

\section{Figura 1}

Grupo de estudiantes con su caja negra y el organizador-guía sobre la mesa

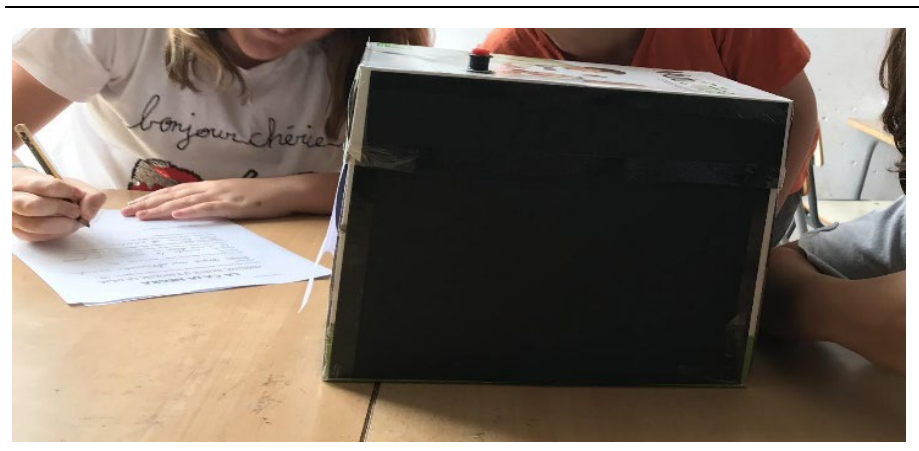

Nota. En su parte superior, la caja tiene un pequeño visor y un interruptor.

Las actividades de aprendizaje (dirigidas a lograr los objetivos planteados) en el juego son: practicar/participar en discusiones responsables y constructivas entre iguales, elaborar comprensión desde los datos observacionales propios y de los demás, animarse para abordar conceptos nuevos o interpretaciones erróneas, compartir la comprensión del contenido y alentar y modelar las habilidades de la investigación científica que caracterizan la práctica realizada, tales como el pensamiento y la argumentación, la curiosidad, el escepticismo y la apertura a nuevas hipótesis, ideas y datos. 
El docente construye una caja cerrada (negra) con objetos dentro y propone a sus estudiantes averiguar el contenido de la caja, mediante el desarrollo de diversas actividades pautadas (individuales, de grupo clase y de grupos pequeños), tales como explorar la caja, escribir los datos observados, buscar pruebas, razonar y elaborar sobre datos y pruebas, proponer hipótesis y explicaciones, elaborar y discutir explicaciones que respalden los datos, y sacar, justificar y argumentar válidamente sus conclusiones. Para ello, pueden manipular la caja, sin romperla o abrirla (inicialmente), para hacer observaciones y experimentar con sus sentidos en grupo pequeño; finalmente, toda la clase pone en común las respuestas de los grupos para discutir y acordar conclusiones globales. Los estudiantes son el centro y el plan debe darles oportunidades para construir sus propios argumentos, datos y modelos, y para estimular su toma de decisiones y su pensamiento creativo.

\section{Resultados}

Los datos y resultados del proceso de investigación-acción con base en las actividades del proyecto de cajas negras son todos los productos elaborados y desarrollados por los maestros en formación para la enseñanza, que comprenden desde el plan de las lecciones hasta la evaluación de la actividad, y los resultados de aprendizaje logrados por el alumnado con el juego, cuyo análisis se presenta como hitos de logro de los objetivos del estudio (Figura 1).

\subsection{La secuencia de enseñanza para el juego de caja negra}

El primer producto de formación del profesorado fue el diseño de la SEA por los maestros para guiar la aplicación de aula (tabla 1), y cuyo patrón de diseño es el modelo didáctico de las 7E (extraer, enganchar/envolver, explicar, explorar, elaborar, extender, evaluar). Las tareas de los maestros implicaron la adaptación a sus estudiantes del juego, sus contenidos, las explicaciones y la pedagogía de las 7E. El plan general prevé plantear al alumnado algunas preguntas iniciales para extraer sus ideas previas y motivarles (¿Cómo trabaja la comunidad científica? ¿Cómo es una investigación científica?) y, después, desarrollar con las cajas negras actividades individuales, en grupos pequeños y en el grupo clase, tales como explorar, elaborar los datos observados, proponer explicaciones, argumentar y discutir las explicaciones y los datos de apoyo, y extender y evaluar las conclusiones. Una actividad final debate las respuestas de los grupos para llegar a una conclusión final de aula.

Los elementos generales de la SEA de cajas negras incluyen la descripción general, los estándares de aprendizaje, las relaciones del juego con los contenidos curriculares, la conexión entre las pruebas, la explicación científica y los modelos teóricos y las actividades del alumnado. La descripción general de la SEA se justificó así: la ciencia difiere de otras formas de conocimiento en que la comunidad científica usa exigentes estándares empíricos, argumentos lógicos y escepticismo, para lograr la mejor explicación posible sobre los hechos observados. El maestro utiliza la caja negra para involucrar a los alumnos en la actividad de búsqueda de explicaciones basadas en pruebas a la pregunta (¿qué hay dentro de la caja?) y solicitando al alumnado justificar sus observaciones, propuestas y respuestas.

Otro resultado a lograr con el juego de cajas negras es el desarrollo de la dimensión epistémica del bloque 1 del currículo de ciencias para educación primaria sobre la actividad científica: las explicaciones científicas se basan en pruebas, desarrollan argumentos y conclusiones consistentes y 
utilizan conocimiento científico (principios, modelos y teorías). La competencia de la alfabetización científica desarrollada en el alumnado con esta SEA es la coordinación lógica y razonada entre pruebas, explicaciones y conclusiones, que implica el logro de los estándares de aprendizaje siguientes, relativos al pensamiento científico:

- Desarrollar destrezas de pensamiento e investigación (observar, medir, clasificar, comprobar, razonar, argumentar, diseñar, ...)

- Crear hipótesis reflexivas y precisas, basadas en observaciones y conocimientos

- Argumentar y hacer predicciones con pruebas

- Entender el método científico como un proceso plural (a veces, cíclico)

- Vincular la investigación científica y las cuestiones sociales

- Desarrollar actitudes y disposiciones propias de la práctica científica (curiosidad, escepticismo, apertura a nuevas ideas y datos, etc.)

- Practicar y participar en debates colectivos, responsables y constructivos

- Cooperar para compartir los contenidos y la comprensión de los contenidos

- Afrontar con seriedad intelectual las ideas y los conceptos, nuevos y erróneos

Tabla 1

Desarrollo de la secuencia de enseñanza-aprendizaje sobre el proyecto de experimento con una caja negra, estructurada según el modelo didáctico de $7 E$

\begin{tabular}{|c|c|c|c|}
\hline Tiempo & ACTIVIDADES (Estudiantes / Profesorado) & $\begin{array}{l}\text { Metodología / } \\
\text { organización }\end{array}$ & $\begin{array}{l}\text { Materiales / } \\
\text { Recursos }\end{array}$ \\
\hline \multicolumn{4}{|c|}{ ENGANCHAR Introducción-motivación } \\
\hline $20^{\prime}$ & $\begin{array}{c}\text { Se presenta a los alumnos la caja y se les motiva a } \\
\text { intentar adivinar qué habrá dentro. Se hace especial } \\
\text { hincapié en que todas las respuestas han de ser } \\
\text { justificadas. (¿Por qué?) }\end{array}$ & Grupo clase & Caja \\
\hline \multicolumn{4}{|c|}{ EXTRAER Conocimientos previos } \\
\hline $20^{\prime}$ & $\begin{array}{l}\text { Responden la pregunta / ¿Cuántas cosas usas } \\
\text { cotidianamente sin conocer lo que tienen dentro? }\end{array}$ & Grupo clase & \\
\hline \multicolumn{4}{|c|}{ EXPLORAR, EXPLICAR, ELABORAR } \\
\hline $45^{\prime}$ & $\begin{array}{c}\text { PRIMERA FASE (observan, mueven y escuchan la caja) } \\
\text { ¿Creéis que todos los elementos que hay en su } \\
\text { interior son iguales? ¿Por qué? } \\
\text { ¿Crees que todos los elementos tienen la misma } \\
\text { masa? ¿Por qué? } \\
\text { ¿Ruedan los elementos que hay en el interior? } \\
\text { Si la respuesta es sí ¿qué forma geométrica tiene } \\
\text { (esfera, cono, cilindro...)? } \\
\text { ¿Cuántos objetos creéis que hay en total? } \\
\text { ¿Cómo lo podrías comprobar? } \\
\text { ¿Qué creéis que hay dentro? ¿Por qué? } \\
\text { ¿Cómo podríais comprobarlo? }\end{array}$ & $\begin{array}{l}\text { Estudiantes en pequeños } \\
\text { grupos cooperativos. } \\
\text { Responden las preguntas. }\end{array}$ & $\begin{array}{c}\text { Caja negra } \\
\text { (contiene tapones } \\
\text { de plástico, de } \\
\text { corcho y pelotas de } \\
\text { poliuretano). } \\
\text { Guía / organizador } \\
\text { con las preguntas a } \\
\text { responder } \\
\text { (colector de datos } \\
\text { de la actividad) }\end{array}$ \\
\hline
\end{tabular}


Formar, enseñar y aprender sobre la práctica científica en primaria con un juego de cajas negras

\begin{tabular}{|c|c|c|c|}
\hline Tiempo & ACTIVIDADES (Estudiantes / Profesorado) & $\begin{array}{l}\text { Metodología / } \\
\text { organización }\end{array}$ & $\begin{array}{c}\text { Materiales / } \\
\text { Recursos }\end{array}$ \\
\hline $50^{\prime}$ & $\begin{array}{c}\text { EXPLORAR, EXPLICAR, ELABORAR } \\
\text { SEGUNDA FASE } \\
\text { ¿LOs elementos que hay en su interior son iguales? } \\
\text { ¿Cuántos tipos de objetos diferentes puedes } \\
\text { observar? } \\
\text { ¿Ahora crees que todos los elementos tienen la } \\
\text { misma masa? } \\
\text { ¿Cómo lo podrías comprobar? } \\
\text { ¿Qué formas geométricas se distinguen en los } \\
\text { objetos de las cajas (esfera, cono cilindro...) } \\
\text { ¿Cuántos objetos creéis que hay en total? } \\
\text { ¿Cómo podríais comprobarlo? } \\
\text { ¿Qué hay dentro? }\end{array}$ & $\begin{array}{l}\text { Los estudiantes pulsan el } \\
\text { botón y pueden mirar por } \\
\text { el agujero al interior de la } \\
\text { caja; responden las } \\
\text { preguntas. }\end{array}$ & $\begin{array}{c}\text { Un circuito } \\
\text { eléctrico con un } \\
\text { LED, que se } \\
\text { enciende pulsando } \\
\text { un botón, y un } \\
\text { agujero por el cual } \\
\text { se observa el } \\
\text { interior un poco. }\end{array}$ \\
\hline $50^{\prime}$ & $\begin{array}{c}\text { EXTENDER, ELABORAR } \\
\text { TERCERA FASE } \\
\text { ¿Qué hay dentro? } \\
\text { ¿Son iguales todos los objetos que hay en el interior? } \\
\text { ¿Cuántos objetos hay en total? } \\
\text { ¿Cuántos tipos de objetos diferentes puedes } \\
\text { observar? } \\
\text { Comprueba la masa de cada objeto con la balanza. } \\
\text { ¿Tienen diferente masa? (Completar tabla) } \\
\text { Clasifica los objetos según su forma geométrica. } \\
\text { (Completar tabla) }\end{array}$ & $\begin{array}{l}\text { Los estudiantes abren la } \\
\text { caja y comprueban sus } \\
\text { hipótesis, respondiendo } \\
\text { las preguntas y } \\
\text { completando las tablas } \\
\text { de la guía con datos }\end{array}$ & $\begin{array}{l}\text { Los estudiantes } \\
\text { pesan los objetos } \\
\text { con una balanza. }\end{array}$ \\
\hline \multicolumn{4}{|c|}{$\begin{array}{l}\text { EXPLICAR Actitudes } \\
\end{array}$} \\
\hline $20^{\prime}$ & $\begin{array}{l}\text { Mentalidad abierta, curiosidad, veracidad (no } \\
\text { trampas, no mentiras...), pensar preciso y profundo, } \\
\text { argumentar conclusiones, imaginación y creatividad... }\end{array}$ & Transversal a actividades & \\
\hline \multicolumn{4}{|c|}{ EVALUAR } \\
\hline $50^{\prime}$ & $\begin{array}{c}\text { Cada grupo EXPONE oralmente sus resultados. } \\
\text { Auto-evalúan el número de aciertos y errores. } \\
\text { Co-evaluación: se extraen conclusiones y propuestas } \\
\text { de mejora. }\end{array}$ & Discusión en grupo clase & \\
\hline
\end{tabular}

Además, los profesores sugieren algunas preguntas adicionales, como actividades de orientación complementarias para elaborar y extender la reflexión del alumnado:

- ¿Qué preguntas tienes acerca de la caja negra?

- ¿Qué queremos decir con pruebas?

- ¿En qué se parece y se diferencia una explicación basada en pruebas de otras explicaciones?

- ¿ ¿En qué se parece y se diferencia el proyecto de la caja negra y una investigación científica?

- ¿Diferentes investigaciones científicas pueden requerir diferentes enfoques?

\subsection{Resultados cualitativos de las actividades de aula}

Como ejemplificación de los procesos de práctica científica planificados para la enseñanza en el aula, los siguientes apartados refieren brevemente algunos resultados cualitativos desarrollados por uno de los maestros. Su SEA del juego de la caja negra planteaba tres fases de actividades, cada una permitiendo un mayor acceso al interior de la caja (tabla 1). 


\section{Fase 1: Plantea hipótesis (observa, mueve y escucha la caja)}

Los estudiantes observan y mueven la caja cerrada, para obtener datos primarios de observación visual-correlacionar movimiento con sonidos y tacto-, táctil-interpretar los impactos de los objetos contra las paredes-, sonora -inferir conclusiones de los sonidos producidos por los choques-, y, eventualmente, olfativa -olores provenientes de los objetos-. Con los datos de estas observaciones los estudiantes responden las preguntas planteadas para esta primera fase (tabla 2).

\section{Tabla 2}

Hipótesis planteadas por tres grupos de estudiantes respecto al contenido de la caja negra como resultado de sus observaciones

\begin{tabular}{|c|c|c|c|}
\hline Fase 1: Preguntas & Grupo A & Grupo B & Grupo C \\
\hline $\begin{array}{l}\text { 1. ¿Creéis que todos los } \\
\text { elementos que hay en su } \\
\text { interior son iguales? }\end{array}$ & No & No & No \\
\hline 2. ¿ंPor qué? & $\begin{array}{l}\text { Porque hay diferentes } \\
\text { sonidos }\end{array}$ & $\begin{array}{l}\text { Porque cuando se } \\
\text { chocan contra la pared } \\
\text { no suena igual }\end{array}$ & $\begin{array}{l}\text { Porque cuando impacta } \\
\text { algunas suenan más } \\
\text { fuerte y otras más ligeras }\end{array}$ \\
\hline $\begin{array}{l}\text { 3. ¿Crees que todos los } \\
\text { elementos tienen la misma } \\
\text { masa? }\end{array}$ & No & No & No \\
\hline 4. ¿ंPor qué? & $\begin{array}{l}\text { Porque cuando la } \\
\text { mueves y pones las } \\
\text { manos debajo se nota }\end{array}$ & $\begin{array}{l}\text { Porque las que pesan } \\
\text { poco suenan mucho, y } \\
\text { las que pesan más } \\
\text { suenan menos }\end{array}$ & $\begin{array}{l}\text { Porque cuando caen } \\
\text { algunas suenan fuerte } y \\
\text { otras no }\end{array}$ \\
\hline $\begin{array}{l}\text { 5. ¿Ruedan los elementos que } \\
\text { hay en el interior? }\end{array}$ & Sí ruedan & No & No \\
\hline $\begin{array}{l}\text { 6. Si la respuesta es sí ¿de qué } \\
\text { cuerpo geométrico creéis } \\
\text { que tiene forma (esfera, } \\
\text { cono cilindro...)? }\end{array}$ & Tiene forma de esfera & & $\begin{array}{l}\text { Algunas son prismas y } \\
\text { otras cilindros }\end{array}$ \\
\hline $\begin{array}{l}\text { 7. ¿Cuántos objetos creéis que } \\
\text { hay en total? }\end{array}$ & Aproximadamente 16 & Entre 10 y 20 & Entre 10 y 15 \\
\hline $\begin{array}{lll}\text { 8. ¿Cómo lo podrías } \\
\text { comprobar? }\end{array}$ & Abriendo la caja & $\begin{array}{l}\text { Abriéndola y contando } \\
\text { los objetos }\end{array}$ & $\begin{array}{l}\text { Contando todos los } \\
\text { impactos }\end{array}$ \\
\hline 9. ¿Qué creéis que hay dentro? & Lápices y pelotitas & $\begin{array}{l}\text { Piezas de Lego, piedras y } \\
\text { quizás pelotas }\end{array}$ & $\begin{array}{l}\text { De madera con formas } \\
\text { de cilindros y de prismas }\end{array}$ \\
\hline 10. ¿Por qué? & Porque lo escuchamos & Por el ruido & Por el sonido del impacto \\
\hline $\begin{array}{l}\text { 11. ¿Cómo } \\
\text { comprobarlo? }\end{array}$ & Abriendo la caja & $\begin{array}{l}\text { Abriendo la caja y } \\
\text { mirando }\end{array}$ & Abriendo la caja \\
\hline
\end{tabular}

Las hipótesis planteadas por el alumnado son ampliamente coincidentes en las respuestas dadas a las tres primeras y las dos últimas cuestiones de la tabla 2. La primera divergencia se observa en las hipótesis relativas a la justificación de la distinta masa que pueden tener los objetos contenidos en la caja (\#4). El grupo A emite una hipótesis imprecisa, el grupo $C$ expresa una hipótesis de proporcionalidad entre intensidad del sonido y masa de los objetos, mientras el grupo $\mathrm{B}$ hace una hipótesis que expresa una relación inversa entre sonido y masa, que resulta singular para la edad de estos alumnos, porque, en cierta medida, es contra-intuitiva. Las restantes cuestiones también ponen de manifiesto las diferencias entre grupos en respuestas e hipótesis, sugiriendo que las observaciones no son siempre obvias. 


\section{Figura 2}

Vista limitada del interior de la caja negra a través de un pequeño agujero en su pared e iluminado por una tenue luz

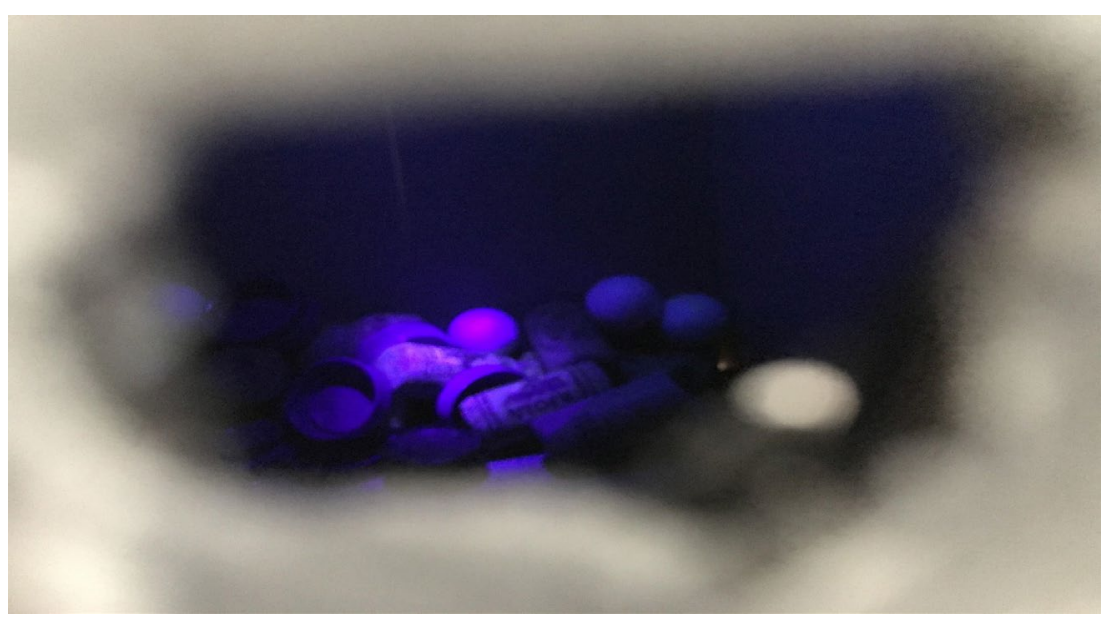

\section{Fase 2: Revisión de hipótesis (encender la luz y observar por un agujero)}

La segunda fase del proyecto somete a revisión las hipótesis emitidas en una nueva fase del contexto de observación: los estudiantes pueden encender una tenue luz dentro de la caja y observar su interior por un agujerito (figura 2). Esta situación emula el descubrimiento de una tecnología que permite a la comunidad científica realizar nuevas observaciones con ayuda de la tecnología aplicada.

Los estudiantes vuelven a observar, mover y escuchar la caja para recoger nuevos datos observacionales que permitan responder a las nuevas preguntas de esta fase. Las hipótesis planteadas en esta situación de observación limitada son mayoritariamente coincidentes (tabla 3). La hipótesis que causa más diferencias entre los grupos es la predicción del número de objetos contenidos en la caja, que oscila entre cinco y veinticinco, y la predicción del número mínimo de objetos (entre cinco y veinte).

\section{Tabla 3}

Hipótesis planteadas por tres grupos de trabajo de estudiantes respecto al contenido de la caja negra como resultado de sus observaciones mediadas por la tecnología (visión del interior de la caja con ayuda de un pequeño agujero y una tenue iluminación)

\begin{tabular}{llllll}
\hline \multicolumn{2}{c}{ Fase 2: Preguntas } & Grupo A & Grupo B & Grupo C \\
\hline $\begin{array}{l}\text { ¿Ahora creéis que todos los No } \\
\text { elementos que hay en su } \\
\text { interior son iguales? }\end{array}$ & No & No \\
\hline $\begin{array}{l}\text { ¿Cuántos tipos de objetos } \\
\text { diferentes puedes observar? }\end{array}$ & Tres & Tres & \\
\hline 3. ¿Ahora crees que todos los & No \\
& $\begin{array}{l}\text { elementos tienen la misma } \\
\text { masa? }\end{array}$ & No & No \\
\hline 4. ¿Cómo lo podrías comprobar? & Cogiéndolos & $\begin{array}{l}\text { Abriéndola y } \\
\text { cogiéndolos }\end{array}$ & $\begin{array}{l}\text { Yendo a coger los } \\
\text { mismos objetos que hay } \\
\text { en el interior de la caja }\end{array}$
\end{tabular}




\begin{tabular}{|c|c|c|c|c|}
\hline & $\begin{array}{l}\text { ¿Qué formas geométricas se } \\
\text { distinguen en los objetos de las } \\
\text { cajas (esfera, cono cilindro...) }\end{array}$ & $\begin{array}{l}\text { Esfera, circunferencias } \\
\text { y cilindro }\end{array}$ & Esferas y cilindros & Cilindro, esfera \\
\hline 6. & $\begin{array}{l}\text { ¿Cuántos objetos creéis que hay } \\
\text { en total? }\end{array}$ & $\begin{array}{l}20-25 \\
\text { aproximadamente }\end{array}$ & Entre 5 y 15 & Entre 10 y 15 \\
\hline 7. & ¿Cómo podríais comprobarlo? & Abriendo la caja & $\begin{array}{l}\text { Abriéndola y } \\
\text { contándolos }\end{array}$ & Abriendo la caja \\
\hline 8. & ¿Qué hay dentro? & $\begin{array}{l}\text { Corchos, tapones y } \\
\text { pelotas de ping-pong }\end{array}$ & $\begin{array}{l}\text { Corchos, tapones y } \\
\text { bolas de ping-pong }\end{array}$ & $\begin{array}{l}\text { Corchos de vino, } \\
\text { tapones de botella y } \\
\text { pelotas pequeñas }\end{array}$ \\
\hline
\end{tabular}

\section{Fase 3. Comprobación de hipótesis (apertura de la caja)}

En la tercera fase los estudiantes pueden abrir la caja y manipular su contenido. Esto permite verificar las hipótesis emitidas y realizar la clasificación de los objetos contenidos en la caja, según su forma geométrica y su masa (medida con una balanza). En los tres grupos los resultados son homogéneos, tanto en la clasificación de los objetos como en las medidas de masa (tabla 4). No obstante, cabe notar como un error experimental -que también se produce en la ciencia- la desviación del grupo B en el recuento del número de objetos de su caja (34).

\section{Tabla 4}

Hipótesis planteadas por tres grupos de trabajo de estudiantes respecto al contenido de la caja negra como resultado de sus observaciones y medidas después de abrir la caja

\begin{tabular}{|c|c|c|c|}
\hline Fase 3: Preguntas & Grupo A & Grupo B & Grupo C \\
\hline ¿Qué hay dentro? & $\begin{array}{l}\text { Corchos, corchos } \\
\text { cortados, pelotas de } \\
\text { corcho y tapones }\end{array}$ & $\begin{array}{l}\text { Corchos, tapones y bolas } \\
\text { de porespan }\end{array}$ & $\begin{array}{l}\text { Tapones de botella, } \\
\text { corchos vino, pelotas de } \\
\text { poliespan }\end{array}$ \\
\hline $\begin{array}{l}\text { ¿Son iguales todos los objetos que } \\
\text { hay en el interior? }\end{array}$ & No & No & No son iguales \\
\hline ¿Cuántos objetos hay en total? & 33 & 34 & 33 \\
\hline $\begin{array}{l}\text { ¿Cuántos tipos de objetos } \\
\text { diferentes puedes observar? }\end{array}$ & 3 & Tres & 3 \\
\hline \multirow[t]{2}{*}{$\begin{array}{l}\text { Comprueba la masa de cada } \\
\text { objeto. ¿Tienen diferente masa? }\end{array}$} & $\begin{array}{l}\text { Tapón medio gramo } \\
\text { Corcho } 8 \mathrm{~g}\end{array}$ & $\begin{array}{l}\text { Tapón medio gramo } \\
\text { Corcho } 8 \mathrm{~g}\end{array}$ & $\begin{array}{l}\text { Tapón medio gramo } \\
\text { Corcho } 8 \mathrm{~g}\end{array}$ \\
\hline & $\begin{array}{l}\text { Bola de poliuretano } \\
\text { medio gramo }\end{array}$ & $\begin{array}{l}\text { Bola de poliuretano } \\
\text { medio gramo }\end{array}$ & $\begin{array}{l}\text { Bola de poliuretano } \\
\text { medio gramo }\end{array}$ \\
\hline \multicolumn{4}{|l|}{$\begin{array}{l}\text { Clasifica los objetos según su forma } \\
\text { geométrica }\end{array}$} \\
\hline \multicolumn{4}{|l|}{ FORMA GEOMÉTRICA } \\
\hline CILINDROS & 13 & 13 & 13 \\
\hline ESFERAS & 5 & 5 & 5 \\
\hline OTROS & 15 & 15 & 15 \\
\hline
\end{tabular}

Finalmente, los grupos exponen al resto de la clase los resultados obtenidos, reflexionando sobre las razones de sus aciertos y fallos en las hipótesis planteadas, al compararlas con los resultados de la observación directa del contenido de la caja. 


\subsection{La evaluación formativa de los maestros}

En los procesos de investigación-acción, los docentes son los investigadores de su propia práctica, de modo que la formación y desarrollo profesional de los docentes para enseñar conocimientos epistémicos se completó con una sesión conjunta, donde los maestros compartieron sus experiencias sobre la aplicación de la secuencia de enseñanza-aprendizaje de cajas negras y evaluaron las actividades y resultados logrados con todo el proyecto.

Los tres maestros desarrollaron lecciones diferentes entre sí para adaptarse las necesidades de sus estudiantes y al enfoque del curso de cada uno. Los resultados expuestos anteriormente corresponden al proyecto de uno de los maestros; los proyectos de los otros dos maestros se exponen sucintamente a continuación para remarcar su diversidad.

Uno construyó una única caja dividida en tres partes que contenían una placa base de ordenador, monedas y diversos componentes digitales. Los grupos de estudiantes investigaron la caja y emitieron sus hipótesis con distintas instrucciones; un primer grupo emitió hipótesis sin tocar la caja; el segundo grupo pudo mover la caja; el grupo observó el interior de la caja a través de dos pequeños agujeros practicados en ella. Los grupos pusieron en común sus hipótesis, reflexionaron sobre ellas y acordaron una propuesta común, antes de abrir la caja y verificar sus hipótesis, predicciones e ideas elaboradas (figura 3).

\section{Figura 3}

Grupo de estudiantes del segundo proyecto trabajando con su caja negra

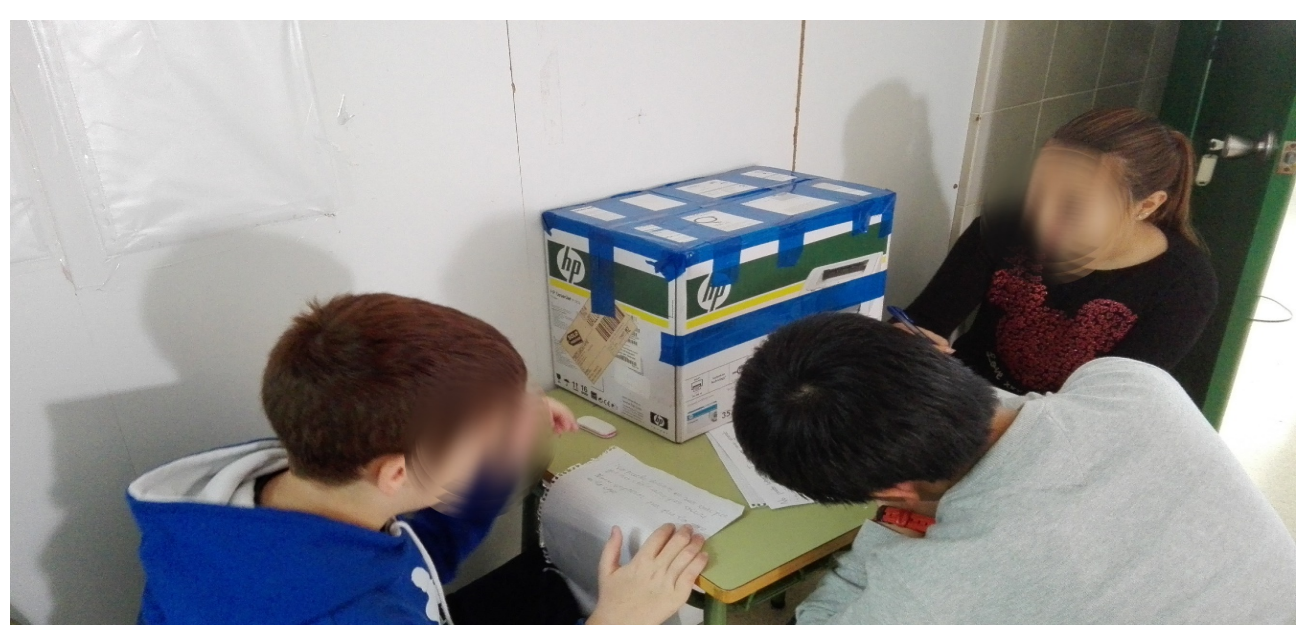

El tercer proyecto desarrolló una fase inicial donde los estudiantes se constituyen en grupos de investigación formales, adoptando incluso un nombre-100tíficos, Laboratorio Einstein y Laboratorio Mikolos-. Después, cada grupo realiza las actividades: medir las dimensiones de la caja, añadir en las hipótesis la práctica del razonamiento al absurdo -objetos excluidos que no podría contener la caja por sus dimensiones- y comunicar por escrito las hipótesis y conclusiones elaboradas, con base en los sonidos de los objetos al mover la caja y relacionarlos con los sonidos musicales, asignatura donde se aplicó la actividad (figura 4). 


\section{Figura 4}

Portada de la comunicación escrita sobre la caja negra presentada por el grupo de trabajo autodenominado Laboratorio Einstein

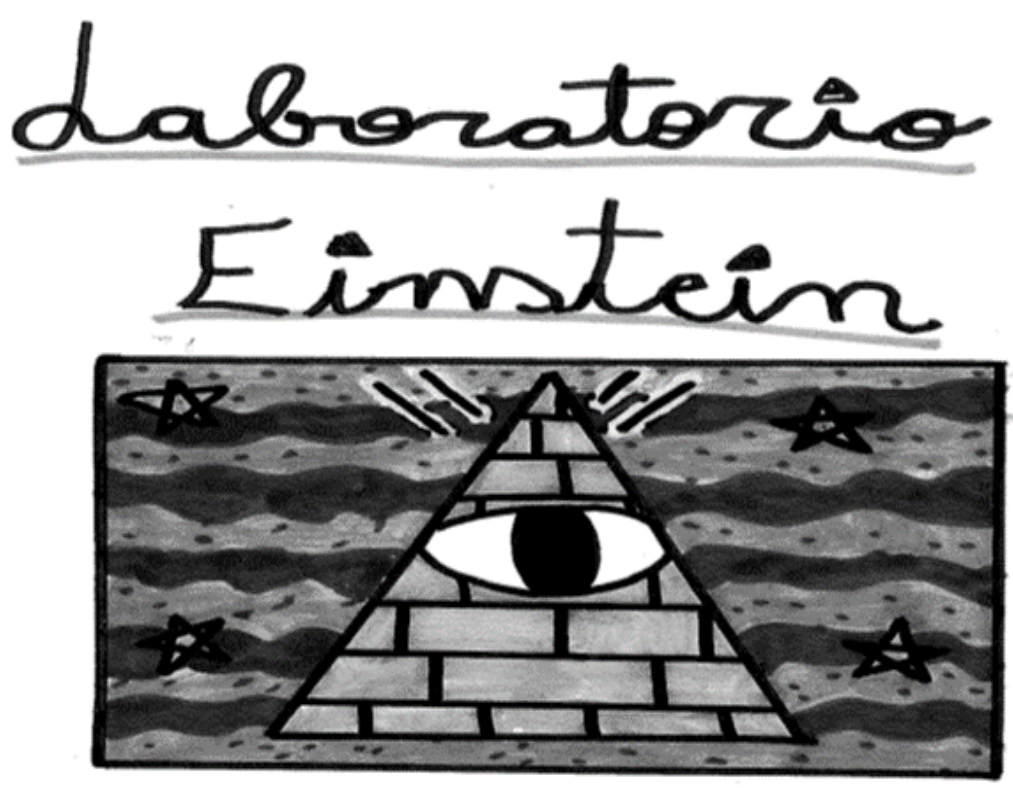

Los tres maestros valoraron positivamente la experiencia porque consideraron que habían logrado los objetivos básicos de su formación: diseñar los elementos didácticos de la lección, aplicar el juego de cajas negras en clase y lograr el éxito de la experiencia entre los estudiantes, ya que completaron sus tareas satisfactoriamente, mantuvieron el interés durante su aplicación y aportaron formas aceptables de argumentación y razonamiento.

La autoevaluación positiva de los maestros se manifestó en diversas expresiones de satisfacción con la experiencia; he aquí dos extractos:

M1. La experiencia me pareció muy positiva en su conjunto, ya que ha puesto en valor pequeñas actividades simples, que pueden ser herramientas mucho más útiles de lo que parecen a primera vista.

M2. ... trabajar actividades creíbles del mismo estilo que enfrentamos en la vida diaria es una de las cosas que he encontrado más estimulantes e interesantes.

Además de las valoraciones positivas generales, los maestros sacaron conclusiones más específicas y críticas. Los tres maestros coinciden que al inicio de la actividad con la caja negra debieron dedicar esfuerzos y tiempo a frenar la impulsividad del alumnado, que producía respuestas irreflexivas y rápidas a las preguntas, reiterando que el juego requiere calma para pensar, elaborar y justificar interpretaciones razonadas, basadas en los datos, porque conducen probablemente a una hipótesis o predicción precisa y válida sobre el contenido de la caja. Algunos comportamientos de los estudiantes se identificaron así:

M3. La alta impulsividad acrítica inicial del alumnado para lanzar en juego la primera idea que se les venía a la boca se redujo progresivamente al aumentar la reflexión sobre las pruebas. 
M2. Los estudiantes aceptaron espontáneamente todas las opiniones, a pesar de ser corregidas o descartadas más tarde.

M3. Los estudiantes no lucharon ni se pelearon para imponer sus puntos de vista personales; más bien aceptaron y trataron de llegar a un consenso a pesar de tener ideas muy diferentes.

M1. Los estudiantes trataron de cambiar y ajustar sus puntos de vista a los hechos, especialmente cuando surgieron nuevas pruebas a lo largo de los intercambios cooperativos y las discusiones.

Las conclusiones anteriores sugieren una positiva y progresiva acomodación de los procesos de autorregulación y meta-cognición a lo largo de las actividades; estos procesos son elementos esenciales del pensamiento crítico, porque contribuyen a aumentar el autocontrol del alumnado en su trabajo cooperativo de creación y discusión de ideas, para resolver el reto planteado con la caja y las sucesivas justificaciones del grupo sobre las decisiones adoptadas. Los maestros decidieron continuar experimentando el curso próximo con otro juego, que constituye la prueba más evidente de la eficacia percibida de la experiencia global.

\section{Discusión}

Este estudio propone una respuesta a las dificultades generales para enseñar y aprender sobre la práctica científica - deficiente formación del profesorado, reticencia a enseñarla explícitamente y carencia de recursos educativos- $y$, en el caso de la educación primaria, el menor desarrollo cognitivo del alumnado y su presencia menos asidua en la literatura. El juego de caja negra ofrece un recurso sencillo y flexible, adaptable al nivel del alumnado, que presenta explícitamente la práctica científica y constituye un instrumento de formación del profesorado, desde una perspectiva de investigación-acción sobre la práctica educativa, diferente a la habitual participación en un curso de formación sobre métodos científicos.

Lo fundamental de la experiencia es lograr planificar y enseñar con eficacia contenidos epistémicos mediante un juego serio de caja negra: el profesorado se apropia y forma con el nuevo recurso didáctico, es decir, planifica la lección, la aplica a sus estudiantes y la evalúa, a través de un proceso de investigaciónacción sobre su práctica educativa. La satisfacción del profesorado con su práctica docente se basa en apreciar la adecuación, flexibilidad y eficacia del recurso didáctico para desarrollar la enseñanza de los contenidos epistémicos y para adaptarse a las necesidades del alumnado, fomentando así su motivación y su interés por aprender temas cognitivamente complejos (Abd-el-Khalick y Akerson, 2009).

La investigación-acción sobre la práctica con el juego es deliberadamente sencilla, para permitir una formación del profesorado equilibrada, segura y tranquila, de modo que puedan mejorar gradual, asequible y sosteniblemente la complejidad del desarrollo profesional en estos difíciles temas, a través de su experiencia actual gratificante (y futura con otros juegos). De hecho, los participantes mostraron satisfacción, y propusieron continuar enseñando con nuevos juegos para profundizar en el conocimiento didáctico del contenido epistémico (Acevedo-Díaz y García-Carmona, 2016; Vázquez-Alonso y ManasseroMas, 2017).

El juego de caja negra promueve explícitamente el desarrollo de habilidades transversales de aprendizaje, en este caso, relacionados con las destrezas de pensamiento científico y crítico, tales como hacer (y responder) preguntas, buscar datos, identificar regularidades y patrones, tomar decisiones, crear hipótesis, predecir conclusiones, compartir ideas, argumentar con base en pruebas, discutir resultados y comunicar 
conclusiones. Todas ellas, además de enseñar NdC/CE contribuyen a las competencias educativas generales (NGSS, 2013; Vázquez-Alonso y Manassero-Mas, 2018).

Las dificultades de aprendizaje que plantean las complejidades de la investigación científica y el conocimiento $\mathrm{NdC} / \mathrm{CE}$ son superadas por su simplificación en las situaciones analógicas del juego de caja negra. Esta crucial ventaja didáctica podría objetarse, ciertamente, argumentando que el juego ofrece solo un modelo limitado y parcial de las prácticas científicas. Sin embargo, los intangibles logrados con las cajas negras superan la relativa limitación de su representación, en primer lugar, porque estos aprendizajes, tal vez, no se podrían lograr de otra forma; el juego hace accesible el complejo conocimiento NdC/CE a los profesores para enseñarlo $-\mathrm{y}$ al alumnado para aprender-, logro que compensa sus potenciales limitaciones, pues enseñarlo siempre es mejor que no enseñarlo; en segundo lugar, el juego permite desarrollar destrezas de pensamiento científico a profesores y estudiantes, que resultan claves para una alfabetización científica competente, al centrarse en el núcleo de la misma (argumentar con base en pruebas) y evitar distractores que confundan a estudiantes y maestros (Clark et al., 2016); tercero, el recurso del juego trasmite confianza a profesores y estudiantes noveles en su uso al presentar como asequible, útil y práctico el elusivo conocimiento NdC/CE. Finalmente, la satisfacción, las disposiciones y las actitudes favorables logradas en profesores y estudiantes con la experiencia del juego constituyen hitos valiosos que aportan significativamente a un marco contextual (primaria) tradicionalmente relegado en esta línea alfabetizadora. En suma, el positivo balance entre la posible limitación del juego y su capacidad para generar satisfacción, compromiso y confianza en los maestros para enseñar $\mathrm{NdC} / \mathrm{CE}$ y la motivación del alumnado hacia su aprendizaje, constituye una solución innovadora ante la tradicional omisión de estos contenidos en la educación científica (Abd-el-Khalick y Akerson, 2009; Lederman, 2007).

Por otro lado, Wouters et al. (2013) sugieren algunos factores que catalizan positivamente la eficacia del juego para aprender: jugar en grupo cooperativo, suplementar el juego con información de aprendizaje relevante y dedicar múltiples sesiones a la práctica del juego. El primer factor ha sido un elemento determinante para seleccionar el juego cooperativo en esta experiencia -como alternativa al mayor individualismo de los juegos digitales-. Los otros dos factores sugieren sendas propuestas para mejorar la eficacia de los juegos: incrementar el tiempo de práctica y complementarla con ejemplos ilustradores y coherentes con el juego. En este sentido, la eficacia de la caja negra podría mejorarse añadiendo algunos ejemplos de caja negra que han sido cruciales en la historia de la ciencia -el átomo, la pila electroquímica, la fotosíntesis de una planta, los agujeros negros, los ecosistemas, etc.- y múltiples aparatos como telescopios, microscopios, radios, ordenadores, etc. (McComas, 2008).

Cabe destacar también la auto-sostenibilidad de este proyecto de formación e innovación, pues su práctica no demanda requisitos previos ni grandes recursos. Al contrario, la flexibilidad de los juegos permite múltiples adaptaciones sistemáticas para alcanzar logros diversos, tales como enseñar diferentes temas y destrezas, en diferentes niveles educativos y con diferente grado de dificultad. Esta adaptabilidad desarrolla más la auto-confianza, que resulta clave para la continuidad de la formación del profesorado y la enseñanza de estos temas. Finalmente, la sostenibilidad plantea la prospectiva de extender la enseñanza de la práctica científica basada en juegos a todos los niveles del sistema educativo ya que conjuga dos ideas clave y fructíferas: jugar (motivación, confianza) y pensar (crear, argumentar, razonar). Los estudiantes de cualquier edad están motivados para jugar y, además, hay destrezas de pensamiento adecuadas para cada edad; por ello, cualquier juego es potencialmente adaptable a cualquier nivel, desde la educación temprana -usando juegos y destrezas elementales, tales como seguir reglas, plantear preguntas y buscar respuestashasta los niveles superiores -juegos y destrezas creativos y divergentes- para modelizar, verificar, argumentar con base en pruebas, etc. Como demuestra este estudio, el reto es combinar juego y destrezas 
de pensamiento adecuados para cada nivel y edad y para diseñar el conocimiento didáctico del contenido necesario para su enseñanza (Vázquez-Alonso y Manassero-Mas, 2017, 2018).

\section{Organismos colaboradores}

Proyecto EDU2015-64642-R (AEI/FEDER, UE) financiado por la Agencia Estatal de Investigación y el Fondo Europeo de Desarrollo Regional. Proyectos de innovación orientados a la formación inicial del profesorado, convocatoria 2017, IRIE / UIB y Conselleria d'Educació.

\section{Referencias}

Abd-el-Khalick, F., y Akerson, V. (2009). The influence of metacognitive training on preservice elementary teachers' conceptions of nature of science. International Journal of Science Education, 31, 21612184. https://doi.org/10.1080/09500690802563324

Acevedo-Díaz, J. A., y García-Carmona, A. (2016) «Algo antiguo, algo nuevo, algo prestado». Tendencias sobre la naturaleza de la Ciencia en la educación científica. Revista Eureka sobre Enseñanza y Divulgación de las Ciencias, 13(1), 3-19.

https://doi.org/10.25267/rev_eureka_ensen_divulg_cienc.2016.v13.i1.02

Akerson, V. L., Carter, I., Pongsanon, K., y Nargund-Joshi, V. (2019). Teaching and learning nature of science in elementary classrooms. Research-based strategies for practical implementation. Science \& Education, 28, 391-411. https://doi.org/10.1007/s11191-019-00045-1

Akerson, V. L., y Donnelly, L. A. (2010). Teaching nature of science to K-2 students: What understandings can they attain? International Journal of Science Education, 32(1), 97-124. https://doi.org/10.1080/09500690902717283

Akerson, V. L., Nargund-Joshi, V., Weiland, I., Pongsanon, K., y Avsar, B. (2014). What third-grade students of differing ability levels learn about nature of science after a year of instruction. International Journal of Science Education, 36(2), 224-276. https://doi.org/10.1080/09500693.2012.761365

Allchin, D. (2011). Evaluating Knowledge of the Nature of (Whole) Science. Science Education, 95, 518-542. https://doi.org/10.1002/sce.20432

Bunge, M. (1963). Una teoría general de la caja negra. Filosofía de la Ciencia, 30(4), 346-358.

Cartier, J. (2000). Assessment of explanatory models in genetics: insights into students 'conceptions of scientific models. Report no 98-1. University of Wisconsin-Madison.

Clark, D. B., Tanner-Smith, E. E., y Killingsworth S. S. (2016). Digital Games, Design, and Learning: A Systematic Review and Meta-Analysis. Review of Educational Research, 86(1), 79-122. https://doi.org/10.3102\%2F0034654315582065

Cofré, H., Nuñez, P., Santibáñez, D., Pavez, J. M., Valencia, M., y Vergara, C. (2019). A critical review of students' and teachers' understandings of nature of science. Science \& Education, 28, 205-248. https://doi.org/10.1007/s11191-019-00051-3 
Deng, F., Chen, D.-T., Tsai, C.-C., y Chai, C.-S. (2011). Students' Views of the Nature of Science: A Critical Review of Research. Science Education, 95, 961-999. https://doi.org/10.1002/sce.20460

Erduran, S., y Dagher, Z. R. (eds.) (2014). Reconceptualizing the Nature of Science for Science Education. Scientific Knowledge, Practices and Other Family Categories. Springer.

García-Carmona, A., Vázquez, A., y Manassero, M. A. (2011). Estado actual y perspectivas de la enseñanza de la naturaleza de la ciencia: una revisión de las creencias y obstáculos del profesorado. Enseñanza de las Ciencias, 28, 403-412.

Gee, J. P. (2007). What video games have to teach us about learning and literacy. Palgrave Macmillan.

Hassinger-Das, B., Toub, T. S., Zosh, J. M., Michnick, J., Golinkoff, R., y Hirsh-Pasek, K. (2017). More than just fun: a place for games in playful learning. Infancia y Aprendizaje, 40(2), 191-218. https://doi.org/10.1080/02103702.2017.1292684

Hattie, J. (2009). Visible learning: A synthesis of over 800 meta-analyses relating to achievement. Routledge.

Hodson, D. (2008). Towards scientific literacy: A teachers' guide to the history, philosophy and sociology of science. Sense Publishers.

Khadija, E. F., Master, H., y Akerson, V. L. (2015). Using history of science to teach nature of science to elementary students. Science \& Education, 24, 1103-1140. https://doi.org/10.1007/s11191-0159783-5

Lederman, N. G. (2007). Nature of science: past, present, and future. En S. K. Abell y N. G. Lederman (eds.), Handbook of research on science education (pp. 831-879). Lawrence Erlbaum Associates.

Li, M.-C., y Tsai, C.-C. (2013). Game-Based Learning in Science Education: A Review of Relevant Research. Journal of Science Education and Technology, 22, 877-898. https://doi.org/10.1007/s10956-0139436-x

Manassero-Mas, M. A., y Vázquez-Alonso, A. (2019). Conceptualización y taxonomía para estructurar los conocimientos acerca de la ciencia. Revista Eureka sobre Enseñanza y Divulgación de las Ciencias, 16(3), 3104. https://doi.org/10.25267/rev_eureka_ensen_divulg_cienc.2019.v16.i3.3104

Matthews, M. R. (2012). Changing the focus: From nature of science (NOS) to features of science (FOS). En M. S. Khine (ed.), Advances in nature of science research. Concepts and methodologies (pp. 3-26). Springer. https://doi.org/10.1007/978-94-007-2457-0_1

McComas, W. F. (2008). Seeking historical examples to illustrate key aspects of the nature of science. Science \& Education, 17, 249-263. https://doi.org/10.1007/s11191-007-9081-y

McGonigal, J. (2011). Reality is Broken: Why Games Make Us Better and How They Can Change the World. Penguin.

Millar, R. (2006). Twenty First Century Science: insights from the design and implementation of a scientific literacy approach in school science. International Journal of Science Education, 28, 1499-1521. https://doi.org/10.1080/09500690600718344 
MUSE (Modelling for Understanding in Science) (2002). Earth-moon-sun dynamics project. University of Wisconsin-Madison.

NGSS Lead States (2013). Next Generation Science Standards: For States, By States. The National Academies Press.

Osborne, J., Collins, S., Ratcliffe, M., Millar, R., y Duschl, R. (2003). What "ideas-about-science" should be taught in school science? A Delphi study of the expert community. Journal of Research in Science Teaching, 40, 692-720. https://doi.org/10.1002/tea.10105

Quigley, C., Pongsanon, K., y Akerson, V. L. (2011). If we teach them, they can learn: Young students' views of nature of science during an informal science education program. Journal of Science Teacher Education, 22, 129-149. https://doi.org/10.1007/s10972-010-9201-4

Raviolo, A., Ramírez, P., y López, E. A. (2010). Enseñanza y aprendizaje del concepto de modelo científico a través de analogías. Revista Eureka sobre Enseñanza y Divulgación de las Ciencias, 7, 581-612. https://doi.org/10.25267/rev_eureka_ensen_divulg_cienc.2010.v7.i3.01

Roberts, D. (2007). Scientific literacy/science literacy. En S. K. Abell y N. G. Lederman (eds.), Handbook of research on science education (pp. 729-780). Lawrence Erlbaum.

Solís, C., Escriva, I., y Rivero, A. (2015). Una experiencia de aprendizaje por investigación con cajas negras en formación inicial de maestros. Revista Eureka sobre Enseñanza y Divulgación de las Ciencias, 12, 167-177. https://doi.org/10.25267/rev_eureka_ensen_divulg_cienc.2015.v12.i1.11

Squire, K. (2011). Video games and learning: Teaching and participatory culture in the digital age. Teachers College Press.

Vázquez-Alonso, A. y Manassero-Mas, M. A. (2012). La selección de contenidos para enseñar naturaleza de la ciencia y tecnología (parte 1): Una revisión de las aportaciones de la investigación didáctica. Revista Eureka sobre Enseñanza y Divulgación de las Ciencias, 9(1), 2-31. https://doi.org/10.25267/rev_eureka_ensen_divulg_cienc.2012.v9.i1.02

Vázquez-Alonso, A., y Manassero-Mas, M. A. (2017). Juegos para enseñar la naturaleza del conocimiento científico y tecnológico. Educar, 53(1), 149-170. https://doi.org/10.5565/rev/educar.839

Vázquez-Alonso, Á., y Manassero-Mas, M. A. (2018). Más allá de la comprensión científica: educación científica para desarrollar el pensamiento. Revista Electrónica de Enseñanza de las Ciencias, 17(2), 309-336.

Vázquez, Á. (Ed.), Rodríguez, A. M. y Vega, A. P. (2017). Investigación-acción y enseñanza de temas de naturaleza de la ciencia. Editorial Académica Española.

Vesterinen, V-M., Manassero-Mas, M-A., y Vázquez-Alonso, Á. (2014). History, Philosophy, and Sociology of Science and Science-Technology-Society Traditions in Science Education: Continuities and Discontinuities. En M. R. Matthews (ed.), International Handbook of Research in History, Philosophy and Science Teaching (pp. 1895-1925). Springer. https://doi.org/10.1007/978-94-007-7654-8_58

Whitebread, D. (2012). The importance of play. Toy Industries of Europe (TIE). 
Wouters, P., van Nimwegen, C., van Oostendorp, H., y van der Spek, E. D. (2013). A Meta-Analysis of the Cognitive and Motivational Effects of Serious Games. Journal of Educational Psychology, 105, 249265. https://doi.apa.org/doi/10.1037/a0031311 